\title{
QUASI-INJECTIVE MODULES SATISFYING CERTAIN RELATIVE FINITENESS CONDITIONS
}

\author{
TATSUO IZAWA
}

(Received 28 August 28 1986)

Communicated by R. Lidl

Dedicated to the memory of Professor Akira Hattori

\begin{abstract}
We study the endomorphism ring of a quasi-injective right $R$-module $Q$ such that $R$ satisfies certain finiteness conditions relative to $Q$. And we are concerned with a module ${ }_{S} \operatorname{Hom}_{R}(M, Q)$, where $S$ is the endomorphism ring of $Q_{R}$
\end{abstract}

1980 Mathematics subject classification (Amer. Math. Soc.): primary 16 A 52; secondary 16 A 65.

\section{Introduction}

Endomorphism rings of $\Sigma$ (respectively $\Delta$ )-(quasi-)injective modules over an associative ring with identity have been studied mainly by Faith and Năstăsescu (refer to [4], [10], [2], and so on). An injective right $R$-module $Q$ is said to be $\Sigma$ (respectively $\Delta$ )-injective if the lattice of all $Q$-closed right ideals of $R$, that is, $C_{Q}(R)=\left\{I_{R} \subseteq R_{R} \mid R / I\right.$ is $Q$-torsionless $\}$ is noetherian (respectively artinian). Faith has shown in [4] that the endomorphism ring of a finitely generated $\Sigma$ (respectively $\Delta$ )-injective right $R$-module is a right perfect (respectively a left artinian) ring. Moreover, Năstăsescu has shown in [10] that (1) the endomorphism ring $\operatorname{End}\left(Q_{R}\right)$ of a $\Sigma$ (respectively $\Delta$ )-injective right $R$-module $Q$ which has a finitely generated $R$-submodule $Q^{\prime}$ such that $\operatorname{Hom}_{R}\left(Q / Q^{\prime}, Q\right)=(0)$ (in particular, of a finitely generated $\Sigma$ (respectively $\Delta$ )-injective right $R$-module

(C) 1988 Australian Mathematical Society $0263-6115 / 88 \$ A 2.00+0.00$ 
$Q$ ), is a semi-primary (respectively a left artinian) ring, and (2) if $Q$ is a noetherian or artinian, $\Delta$-injective right $R$-module, then $\operatorname{End}\left(Q_{R}\right)$, the endomorphism ring of $Q_{R}$, is a left artinian ring and $\operatorname{Biend}\left(Q_{R}\right)$, the biendomorphism ring of $Q_{R}$, is a right artinian ring.

In the present paper we shall generalize those results to the case where $Q_{R}$ is quasi-injective. For this purpose we shall introduce the concepts of $Q$-noetherian, $Q$-artinian and $Q$-finitely generated modules with respect to any right $R$-module $Q$. And we shall show that when $Q$ is a quasi-injective right $R$-module with $S=\operatorname{End}\left(Q_{R}\right)$ and $M$ is a $Q$-finitely generated (in particular, finitely generated) right $R$-module such that $Q$ is $M$-injective, then (1) if $R_{R}$ is $Q$-noetherian, ${ }_{s} \operatorname{Hom}_{R}(M, Q)$ is coperfect (Theorem 4.1), (2) if $R_{R}$ is $Q$-artinian, $s_{S} \operatorname{Hom}_{R}(M, Q)$ is noetherian (Theorem 4.4), and (3) if $R_{R}$ is both $Q$-noetherian and $Q$-artinian, ${ }_{s} \operatorname{Hom}_{R}(M, Q)$ has finite length (Theorem 4.6). As these applications, we shall show that when $Q$ is a quasi-injective, $Q$-finitely generated (in particular, finitely generated) right $R$-module with $S=\operatorname{End}\left(Q_{R}\right)$, then (1) if $R_{R}$ is $Q$-noetherian, then $S$ is a semi-primary ring (Theorem 4.2), (2) if $R_{R}$ is $Q$-artinian, then $S$ is a left noetherian ring (Corollary 4.5), and (3) if $R_{R}$ is both $Q$-noetherian and $Q$-artinian, then $S$ is a left artinian ring (Corollary 4.8). In addition, we shall show that if $Q$ is a noetherian or artinian, quasi-injective right $R$-module such that $R_{R}$ is $Q$-artinian, then $\operatorname{End}\left(Q_{R}\right)$ is a left artinian ring and $\operatorname{Biend}\left(Q_{R}\right)$ is a right artinian ring (Theorems 4.12 and 4.13). In the sequel, in Section 5 we shall be concerned with endomorphism rings of (quasi-)projective, quasi-injective modules satisfying some finiteness conditions.

\section{Preliminaries}

Let $R$ be an associative ring with identity and Mod- $R$ the category of all unital right $R$-modules. For $M, Q \in \operatorname{Mod}-R, M$ is said to be $Q$-torsion if $\operatorname{Hom}_{R}(M, Q)=(0)$, and said to be $Q$-torsionless if $M$ is embeddable in a direct product of copies of $Q$. An $R$-submodule $L$ of $M$ is said to be a $Q$-closed submodule of $M$ if $M / L$ is $Q$-torsionless. The set of all $Q$-closed submodules of $M$ is denoted by $C_{Q}(M)$ throughout this paper. It is well known that $L \in C_{Q}(M)$ if and only if $L=\operatorname{Ann}_{M}\left(\operatorname{Ann}_{M^{*}}(L)\right)$, where $M^{*}=\operatorname{Hom}_{R}(M, Q)$. We set $\tau_{Q}(M)=\operatorname{Ann}_{M}\left(M^{*}\right)=\left\{x \in M \mid f(x)=0\right.$ for all $\left.f \in M^{*}=\operatorname{Hom}_{R}(M, Q)\right\}$ for $M, Q \in \operatorname{Mod}-R$. Clearly, $\tau_{Q}(M)$ is the smallest $Q$-closed submodule of $M$. By setting $L \wedge N=L \cap N$ and $(L \vee N) /(L+N)=\tau_{Q}(M /(L+N))$ for $L, N \in C_{Q}(M)$, we can give a lattice structure to $C_{Q}(M)$. We set $\Psi(Q)=\{M \in \operatorname{Mod}-R \mid Q$ is $M$-injective $\}$ for any $Q \in \operatorname{Mod}-R$. If $Q \in \Psi(Q), Q$ is said to be quasi-injective, and if $\Psi(Q)=\operatorname{Mod}-R, Q$ is injective. The following result is well known. 
LEMMA 2.1. $\Psi(Q)$ is closed under taking submodules, homomorphic images and direct sums.

If $C_{Q}(R)=\left\{I_{R} \subseteq R_{R} \mid I=\operatorname{Ann}_{R}(X)\right.$ for some subset $X$ of $\left.Q\right\}$ satisfies the ACC (respectively DCC), then $Q_{R}$ is said to be a $\Sigma$ (respectively $\Delta$ )-module for any $Q \in$ Mod- $R$. If an (a quasi-)injective right $R$-module $Q$ is a $\Sigma$ (respectively $\Delta$ )-module, $Q$ is said to be $\Sigma$ (respectively $\Delta$ )-(quasi-)injective. For $M \in \operatorname{Mod}-R$, let $\operatorname{End}\left(M_{R}\right)$ denote the endomorphism ring of $M_{R}$ and $\operatorname{Biend}\left(M_{R}\right)$ the biendomorphism ring of $M_{R}$, that is, $\operatorname{Biend}\left(M_{R}\right)=\operatorname{End}(s M)$, where $S=\operatorname{End}\left(M_{R}\right)$. Any homomorphism will be written on the side opposite to the scalars. For $M \in \operatorname{Mod}-R, M^{n}$ denotes the direct sum of $n$-copies of $M_{R}$. The ACC (respectively DCC) denotes the ascending (respectively descending) chain condition.

\section{3. $Q$-noetherian modules and $Q$-artinian modules}

Let $E$ be an injective right $R$-module and $\mathcal{F}=\left\{I_{R} \subseteq R_{R} \mid \operatorname{Hom}_{R}(R / I, E)=\right.$ $(0)\}$. Then $\mathcal{F}$ is a Gabriel topology on $R$ associated with a hereditary torsion theory defined by $E$. In [11], [8] and [1], Nð̌stăsescu-Niţa-Albu have defined and studied $\mathcal{F}$-noetherian and $\mathcal{F}$-artinian modules and rings. In this section we shall define and study $Q$-noetherian and $Q$-artinian modules in case $Q_{R}$ is not necessarily an injective module.

DEFINITIONS. Let $M, Q \in$ Mod- $R$.

(1) $M$ is said to be $Q$-noetherian (respectively $Q$-artinian) if, for each ascending (respectively descending) chain

$$
\left.M_{1} \subseteq M_{2} \subseteq M_{3} \subseteq \cdots \quad \text { (respectively } M_{1} \supseteq M_{2} \supseteq M_{3} \supseteq \cdots\right) \text { ) }
$$

of $R$-submodules of $M$, there exists an integer $k \geq 1$ such that $M_{i+1} / M_{i}$ (respectively $\left.M_{i} / M_{i+1}\right)$ is $Q$-torsion for all $i \geq k$. A ring $R$ is said to be $Q$-noetherian (respectively $Q$-artinian) if $R_{R}$ is $Q$-noetherian (respectively $Q$-artinian).

(2) If $A$ is a non-empty set of $R$-submodules of $M, N \in A$ is said to be a $Q$-maximal (respectively $Q$-minimal) element in $A$ if, for each $N^{\prime} \in A$ such that $N \subseteq N^{\prime}$ (respectively $N^{\prime} \subseteq N$ ), $N^{\prime} / N$ (respectively $N / N^{\prime}$ ) is $Q$-torsion.

(3) $M$ is said to be $Q$-finitely generated if there exists a finitely generated $R$-submodule $M^{\prime}$ of $M$ such that $M / M^{\prime}$ is $Q$-torsion.

If $Q$ is a cogenerator in Mod- $R$, each $Q$-noetherian (respectively $Q$-artinian) module is exactly a noetherian (respectively artinian) module. When $Q$ is an injective right $R$-module cogenerating a hereditary torsion theory associated with a Gabriel topology $\mathcal{F}$, these definitions are identified with those of $\mathcal{F}$-noetherian, $\mathcal{F}$-artinian, $\mathcal{F}$-maximal, $\mathcal{F}$-minimal and $\mathcal{F}$-finitely generated modules in the sense of Nðstăsescu-Niţa-Albu. 
LEMMA 3.1. Let $M \in \Psi(Q)$. If $M$ is $Q$-torison, then $M$ is both $Q$-noetherian and $Q$-artinian.

ProOF. Let $M_{1} \subseteq M_{2} \subseteq M_{3} \subseteq \cdots$ be any ascending chain of $R$-submodules of $M$. Since $M$ is $Q$-torsion, $M / M_{k}$ is $Q$-torsion, too, for all $k \geq 1$. Since $M / M_{k} \in \Psi(Q)$ by Lemma 2.1, every $R$-submodule of $M / M_{i}$, in particular $M_{k+1} / M_{k}$ is $Q$-torsion by [6, Lemma 2.1]. Hence $M$ is $Q$-noetherian. Similarly, $M$ is $Q$-artinian.

LEMMA 3.2. Let us consider the following conditions.

(1) $M$ is $Q$-noetherian.

(2) Each non-empty set of $R$-submodules of $M$ has a $Q$-maximal element.

(3) $C_{Q}(M)$ is a noetherian lattice.

(4) Each $R$-submodule of $M$ is $Q$-finitely generated. Then we have the implications, (1) $\Rightarrow(2)$ and (2) $\Rightarrow(3)$. In addition, if $M \in \Psi(Q)$, all four conditions are equivalent.

Proof. The implications, $(1) \Rightarrow(2)$ and $(2) \Rightarrow(3)$ can be proved in the same manner as in the proof of [2, Proposition 6.1]. Next, assume that $Q$ is $M$-injective.

(3) $\Rightarrow$ (4). Suppose that $M$ has a submodule $N$ which is not $Q$-finitely generated. Choose $x_{1} \in N$ with $x_{1} \neq 0$. Then $N / x_{1} R$ is not $Q$-torsion, and so in particular $N / x_{1} R \neq(0)$. Hence there exists $x_{2} \in N$ such that $x_{1} R \varsubsetneqq$ $x_{1} R+x_{2} R$ and $\left(x_{1} R+x_{2} R\right) / x_{1} R$ is not $Q$-torsion. For, since $N / x_{1} R \in \Psi(Q)$ by Lemma 2.1, $\tau_{Q}\left(N / x_{1} R\right)$ is $Q$-torsion by [6, (2) of Lemma 2.1], and hence $\tau_{Q}\left(N / x_{1} R\right) \varsubsetneqq N / x_{1} R$. Hence for each $x_{2}+x_{1} R \notin \tau_{Q}\left(N / x_{1} R\right)$, there exists $f \in \operatorname{Hom}_{R}\left(N / x_{1} R, Q\right)$ such that $f\left(x_{2}+x_{1} R\right) \neq 0$. So the restriction of $f$ onto $\left(x_{1} R+x_{2} R\right) / x_{1} R$ is not a zero map. Hence $\left(x_{1} R+x_{2} R\right) / x_{1} R$ is not $Q$-torsion. And then, $N /\left(x_{1} R+x_{2} R\right)$ is not $Q$-torsion. Continuing the same argument, we are able to find a strictly ascending chain of $R$-submodules of $M$,

$$
x_{1} R \varsubsetneqq x_{1} R+x_{2} R \varsubsetneqq x_{1} R+x_{2} R+x_{3} R \varsubsetneqq \cdots
$$

such that $N_{k+1} / N_{k}$ is not $Q$-torsion for all $k \geq 1$, where $N_{k}=x_{1} R+x_{2} R+$ $\cdots+x_{k} R$. Let us put $N_{i}^{\prime} / N_{i}=\tau_{Q}\left(M / N_{i}\right)$ for each integer $i$. Then we get an ascending chain of elements of $C_{Q}(M), N_{1}^{\prime} \subseteq N_{2}^{\prime} \subseteq N_{3}^{\prime} \subseteq \cdots$. Suppose $N_{i}^{\prime}=N_{i+1}^{\prime}$ for some $i$. Then $N_{i+1} / N_{i} \subseteq N_{i+1}^{\prime} / N_{i}=N_{i}^{\prime} / N_{i}$. By using Lemma 2.1 and [6, Lemma 2.1], since $N_{i}^{\prime} / N_{i}$ is $Q$-torsion, so is also $N_{i+1} / N_{i}$. This is a contradiction. Consequently, we have $N_{i}^{\prime} \varsubsetneqq N_{i+1}^{\prime}$ for all $i \geq 1$, and which contradicts the assumption (3).

(4) $\Rightarrow$ (1). Let $M_{1} \subseteq M_{2} \subseteq M_{3} \subseteq \cdots$ be an ascending chain of $R$ submodules of $M$. Since $N=\bigcup_{i=1}^{\infty} M_{i}$ is $Q$-finitely generated, then there exist 
$x_{1}, x_{2}, \ldots, x_{n} \in N$ such that $N /\left(x_{1} R+\cdots+x_{n} R\right)$ is $Q$-torsion. On the other hand, there exists an integer $k$ such that $x_{1} R+\cdots+x_{n} R \subseteq M_{k}$, so $N / M_{k+j}$ is $Q$-torsion for all $j \geq 0$. Since $N / M_{k+j} \in \Psi(Q)$ by Lemma 2.1, $M_{k+j+1} / M_{k+j}$ is $Q$-torsion according to [6, (1) of Lemma 2.1]. Hence $M$ is $Q$-noetherian.

LEMMA 3.3. Let us consider the following conditions.

(1) $M$ is $Q$-artinian.

(2) Each non-empty set of $R$-submodules of $M$ has a $Q$-minimal element.

(3) $C_{Q}(M)$ is an artinian lattice.

Then the implications (1) $\Rightarrow(2)$ and (2) $\Rightarrow(3)$ hold. In addition, if $M \in$ $\Psi(Q)$, all three conditions are equivalent.

Proof. The implications, $(1) \Rightarrow(2)$ and $(2) \Rightarrow(3)$ can be proved in the same manner as in the proof of [2, Proposition 6.2]. Next, assume that $Q$ is $M$-injective.

(3) $\Rightarrow$ (1). Let $L_{1} \supseteq L_{2} \supseteq L_{3} \supseteq \cdots$ be a descending chain of $R$-submodules of $M$. Put $L_{i}^{\prime} / L_{i}=\tau_{Q}\left(M / L_{i}\right)$ for each integer $i$. Then we have the descending chain of elements of $C_{Q}(M), L_{1}^{\prime} \supseteq L_{2}^{\prime} \supseteq L_{3}^{\prime} \supseteq \cdots$. By the assumption (3), there exists an integer $k$ such that $L_{k}^{\prime}=L_{k+1}^{\prime}=L_{k+2}^{\prime}=\cdots$. For each $i \geq$ $k, L_{i} / L_{i+1} \subseteq L_{i}^{\prime} / L_{i+1}=L_{i+1}^{\prime} / L_{i+1}=\tau_{Q}\left(M / L_{i+1}\right)$. Since $M / L_{i+1} \in \Psi(Q)$ by Lemma 2.1, $\tau_{Q}\left(M / L_{i+1}\right)$ is $Q$-torsion, and hence so is also $L_{i} / L_{i+1}$ for all $i \geq k$, by using [6, Lemma 2.1$]$.

Proposition 3.4. Let $M, Q \in \operatorname{Mod}-R$, and let

$$
(0) \rightarrow M^{\prime} \stackrel{\psi}{\rightarrow} M \stackrel{\varphi}{\rightarrow} M^{\prime \prime} \rightarrow(0)
$$

be an exact sequence of right $R$-modules. Then, if $M$ is $Q$-noetherian (resp. $Q$ artinian), so are also $M^{\prime}$ and $M^{\prime \prime}$. If $M \in \Psi(Q)$, and if both $M^{\prime}$ and $M^{\prime \prime}$ are $Q$-noetherian (resp. $Q$-artinian), so is also $M$.

Proof. (I) $Q$-noetherian case. The first part of the statement can be proved by the standard discussion. Next, suppose that $M \in \Psi(Q)$ and both $M^{\prime}$ and $M^{\prime \prime}$ are $Q$-noetherian. Let $L$ be an $R$-submodule of $M . \varphi(L)$ has a finitely generated $R$-submodule $N=\sum_{i=1}^{n} z_{i} R$ such that $\varphi(L) / N$ is $Q$-torsion. Choose $x_{i} \in L$ such that $\varphi\left(x_{i}\right)=z_{i}$ for $i=1,2, \ldots, n$. Put $K=\sum_{i=1}^{n} x_{i} R$. On the other hand, $L \cap \psi\left(M^{\prime}\right)$ has a finitely generated $R$-submodule $H=\sum_{j=1}^{m} y_{j} R$ such that $\left(L \cap \psi\left(M^{\prime}\right)\right) / H$ is $Q$-torsion. Then, since $L \cap \varphi^{-1}(N)=\left(L \cap \psi\left(M^{\prime}\right)\right)+K$, we have the exact sequence as follows:

$$
(0) \rightarrow\left(\left(L \cap \psi\left(M^{\prime}\right)\right)+K\right) /(H+K) \rightarrow L /(H+K) \rightarrow \varphi(L) / N \rightarrow(0) .
$$


Since $L /(H+K) \in \Psi(Q)$ by Lemma 2.1, we have the exact sequence,

$$
\begin{aligned}
(0) & \rightarrow \operatorname{Hom}_{R}(\varphi(L) / N, Q) \rightarrow \operatorname{Hom}_{R}(L /(H+K), Q) \\
& \rightarrow \operatorname{Hom}_{R}\left(\left(\left(L \cap \psi\left(M^{\prime}\right)\right)+K\right) /(H+K), Q\right) \rightarrow(0) .
\end{aligned}
$$

Since $\left(\left(L \cap \psi\left(M^{\prime}\right)\right)+K\right) /(H+K)$ is a homomorphic image of $\left(L \cap \psi\left(M^{\prime}\right)\right) / H$, $\left(\left(L \cap \psi\left(M^{\prime}\right)\right)+K\right) /(H+K)$ is $Q$-torsion, too. Hence

$$
\operatorname{Hom}_{R}(\varphi(L) / N, Q)=\operatorname{Hom}_{R}\left(\left(\left(L \cap \psi\left(M^{\prime}\right)\right)+K\right) /(H+K), Q\right)=(0) ;
$$

so $\operatorname{Hom}_{R}(L /(H+K), Q)=(0)$. Therefore $L$ is $Q$-finitely generated. Hence $M$ is $Q$-noetherian.

(II) $Q$-artinian case. The first part can be proved by the standard discussion. Next, suppose that $M \in \Psi(Q)$ and both $M^{\prime}$ and $M^{\prime \prime}$ are $Q$-artinian. let $L_{1} \supseteq$ $L_{2} \supseteq L_{3} \supseteq \cdots$ be a descending chain of $R$-submodules of $M$. Then for the descending chain, $L_{1} \cap \psi\left(M^{\prime}\right) \supseteq L_{2} \cap \psi\left(M^{\prime}\right) \supseteq L_{3} \cap \psi\left(M^{\prime}\right) \supseteq \cdots$, there exists an integer $k$ such that $\left(L_{i} \cap \psi\left(M^{\prime}\right)\right) /\left(L_{i+1} \cap \psi\left(M^{\prime}\right)\right)$ is $Q$-torsion for all $i \geq k$. And, for the descending chain $\varphi\left(L_{1}\right) \supseteq \varphi\left(L_{2}\right) \supseteq \varphi\left(L_{3}\right) \supseteq \cdots$, there exists an integer $k^{\prime}$ such that $\varphi\left(L_{i}\right) / \varphi\left(L_{i+1}\right)$ is $Q$-torsion for all $i \geq k^{\prime}$. Let $n=\max \left\{k, k^{\prime}\right\}$. Then for all $i \geq n$, let us consider the exact sequence as follows:

$$
(0) \rightarrow\left(\left(L_{i} \cap \psi\left(M^{\prime}\right)\right)+L_{i+1}\right) / L_{i+1} \rightarrow L_{i} / L_{i+1} \rightarrow \varphi\left(L_{i}\right) / \varphi\left(L_{i+1}\right) \rightarrow(0) .
$$

Then we have the exact sequence,

$$
\begin{aligned}
(0) & \rightarrow \operatorname{Hom}_{R}\left(\varphi\left(L_{i}\right) / \varphi\left(L_{i+1}\right), Q\right) \rightarrow \operatorname{Hom}_{R}\left(L_{i} / L_{i+1}, Q\right) \\
& \rightarrow \operatorname{Hom}_{R}\left(\left(\left(L_{i} \cap \psi\left(M^{\prime}\right)\right)+L_{i+1}\right) / L_{i+1}, Q\right) \rightarrow(0),
\end{aligned}
$$

because $L_{i} / L_{i+1} \in \Psi(Q)$. Since

$$
\begin{aligned}
\left(\left(L_{i} \cap \psi\left(M^{\prime}\right)\right)+L_{i+1}\right) / L_{i+1} & \cong\left(L_{i} \cap \psi\left(M^{\prime}\right)\right) /\left(\left(L_{i} \cap \psi\left(M^{\prime}\right)\right) \cap L_{i+1}\right) \\
& =\left(L_{i} \cap \psi\left(M^{\prime}\right)\right) /\left(L_{i+1} \cap \psi\left(M^{\prime}\right)\right),
\end{aligned}
$$

$\operatorname{Hom}_{R}\left(\varphi\left(L_{i}\right) / \varphi\left(L_{i+1}\right), Q\right)=\operatorname{Hom}_{R}\left(\left(\left(L_{i} \cap \psi\left(M^{\prime}\right)\right)+L_{i+1}\right) / L_{i+1}, Q\right)=(0)$. Hence $\operatorname{Hom}_{R}\left(L_{i} / L_{i+1}, Q\right)=(0)$, that is, $L_{i} / L_{i+1}$ is $Q$-torsion for all $i \geq n$. Therefore $M$ is $Q$-artinian.

COROLlaRY 3.5. Let $M \in \Psi(Q)$. If $R$ is a $Q$-noetherian (resp. $Q$-artinian) ring, and if $M$ is a $Q$-finitely generated right $R$-module, then $M$ is $Q$-noetherian (resp. Q-artinian).

PrOOF. Since $R$ is $Q$-noetherian (resp. $Q$-artinian), every cyclic right $R$ module is $Q$-noetherian (resp. $Q$-artinian) by Proposition 3.4. By the assumption there exists a finitely generated $R$-submodule $M^{\prime}$ of $M$ such that $M / M^{\prime}$ is $Q$-torsion. Put $M^{\prime}=\sum_{i=1}^{n} x_{i} R$. Then $x_{i} R$ is $Q$-noetherian (resp. $Q$-artinian). 
Since $Q$ is $M$-injective, $\bigoplus_{i=1}^{k} x_{i} R \in \Psi(Q)$ for all integer $k$ such that $1 \leq k \leq n$, by Lemma 2.1. Let us consider the exact sequence,

$$
0 \rightarrow x_{1} R \rightarrow x_{1} R \oplus x_{2} R \rightarrow x_{2} R \rightarrow(0) .
$$

Since $x_{1} R$ and $x_{2} R$ both are $Q$-noetherian (resp. $Q$-artinian), $x_{1} R \oplus x_{2} R$ is $Q$-noetherian (resp. $Q$-artinian) by Proposition 3.4. By the similar discussion, if $x_{1} R+\cdots+x_{k-1} R$ is $Q$-noetherian (resp. $Q$-artinian), the exact sequence

$$
\text { (0) } \rightarrow x_{1} R \oplus \cdots \oplus x_{k-1} R \rightarrow x_{1} R \oplus \cdots \oplus x_{k-1} R \oplus x_{k} R \rightarrow x_{k} R \rightarrow(0)
$$

implies that $x_{1} R \oplus \cdots \oplus x_{k} R$ is $Q$-noetherian (resp. $Q$-artinian) by Proposition 3.4. Thus, we can conclude that $\bigoplus_{i=1}^{k} x_{i} R$ for each $k$, in particular, $\bigoplus_{i=1}^{n} x_{i} R$ is $Q$-noetherian (resp. $Q$-artinian). Next, since the map $\psi: \bigoplus_{i=1}^{n} x_{i} R \rightarrow M^{\prime}$ defined by $\psi\left(x_{1} r_{1}, x_{2} r_{2}, \ldots, x_{n} r_{n}\right)=\sum_{i=1}^{n} x_{i} r_{i}$, is an $R$-epimorphism, $M^{\prime}$ is $Q$ noetherian (resp. $Q$-artinian) by Proposition 3.4. On the other hand, $M / M^{\prime}$ is $Q$-noetherian (resp. $Q$-artinian) by Lemma 3.1. Hence the exact sequence

$$
(0) \rightarrow M^{\prime} \rightarrow M \rightarrow M / M^{\prime} \rightarrow(0)
$$

implies that $M$ is $Q$-noetherian (resp. $Q$-artinian) by Proposition 3.4, as desired.

\section{Quasi-injective module $Q$ such that $R$ is $Q$-noetherian ( $Q$-artinian)}

Let $M \in \operatorname{Mod}-R$. If $C_{M}(R)=\left\{I_{R} \subseteq R_{R} \mid I=\operatorname{Ann}_{R}(X)\right.$ for some subset $X$ of $M\}$ is noetherian (respectively artinian), then $M_{R}$ is said to be a $\Sigma$ (respectively $\Delta$ )-module. If an (a quasi-)injective right $R$-module $Q$ is a $\Sigma$ (respectively $\Delta$ )module, then $Q_{R}$ is said to be a $\Sigma$ (respectively $\Delta$ )-(quasi-)injective module. According to Lemmas 3.2 and 3.3, an injective right $R$-module $Q$ such that $R_{R}$ is $Q$-noetherian (respectively $Q$-artinian) is exactly a $\Sigma$ (respectively $\Delta$ )-injective module. And, a quasi-injective right $R$-module $Q$ such that $R$ is $Q$-noetherian (respectively $Q$-artinian) is $\Sigma$ (respectively $\Delta$ )-quasi-injective. However, a $\Sigma$ (respectively $\Delta$ )-quasi-injective right $R$-module $Q$ does not necessarily satisfy the condition for $R$ to be $Q$-noetherian (respectively $Q$-artinian). In this section we are concerned with a quasi-injective right $R$-module $Q$ such that $R$ is $Q$ noetherian or $Q$-artinian.

THEOREM 4.1. Let $Q$ be a quasi-injective right $R$-module such that $R$ is $Q$-noetherian and $S=\operatorname{End}\left(Q_{R}\right)$. If $M_{R}$ is $Q$-finitely generated (in particular, finitely generated) and $M \in \Psi(Q)$, then ${ }_{S} \operatorname{Hom}_{R}(M, Q)$ is coperfect.

Proof. Since $M$ is a $Q$-finitely generated right module over a $Q$-noetherian ring $R, M$ is $Q$-noetherian by Corollary 3.5. Hence $C_{Q}(M)$ is a noetherian lattice by Lemma 3.2. Therefore ${ }_{S} \operatorname{Hom}_{R}(M, Q)$ is coperfect by [6, Theorem 4.1] or [2, Corollary 4.3$]$. 
THEOREM 4.2. Let $Q$ be a quasi-injective, $Q$-finitely generated (in particular, finitely generated) right $R$-module such that $R$ is $Q$-noetherian. Then $S=\operatorname{End}\left(Q_{R}\right)$ is a semi-primary ring.

ProOF. In this case $C_{Q}(Q)$ is a noetherian lattice. Hence $S$ is a semi-primary ring by [2, Corollary 4.5].

COROLLARY 4.3. If $Q$ is a quasi-injective, $Q$-finitely generated (in particular, finitely generated) right module over a right noetherian ring $R$, then $S=\operatorname{End}\left(Q_{R}\right)$ is a semi-primary ring.

THEOREM 4.4. Let $Q$ be a quasi-injective right $R$-module such that $R$ is $Q$ artinian and $S=\operatorname{End}\left(Q_{R}\right)$. If $M$ is a $Q$-finitely generated (in particular, finitely generated) right $R$-module and $M \in \Psi(Q)$, then ${ }_{S} \operatorname{Hom}_{R}(M, Q)$ is noetherian.

ProOF. Since $M_{R}$ is a $Q$-finitely generated module over a $Q$-artinian ring $R, M_{R}$ is $Q$-artinian by Corollary 3.5. Hence $C_{Q}(M)$ is an artinian lattice by Lemma 3.3. According to [6, Theorem 4.3] or [2, Corollary 4.3], ${ }_{S} \operatorname{Hom}_{R}(M, Q)$ is noetherian if and only if $C_{Q}(M)$ is artinian, as desired.

COROLLARY 4.5. Let $Q$ be a quasi-injective, $Q$-finitely generated (in particular, finitely generated) right $R$-module such that $R$ is $Q$-artinian. Then $S=\operatorname{End}\left(Q_{R}\right)$ is a left noetherian ring.

THEOREM 4.6. Let $Q$ be a quasi-injective right $R$-module such that $R$ is both $Q$-noetherian and $Q$-artinian and $S=\operatorname{End}\left(Q_{R}\right)$. If $M$ is a $Q$-finitely generated (in particular, finitely generated) right $R$-module and $M \in \Psi(Q)$, then ${ }_{s} \operatorname{Hom}_{R}(M, Q)$ has finite length.

PrOOF. By Theorems 4.1 and 4.4, ${ }_{S} \operatorname{Hom}_{R}(M, Q)$ is coperfect and noetherian. Therefore $s_{S} \operatorname{Hom}_{R}(M, Q)$ has finite length.

COROLLARY 4.7. Let $Q$ be a $\Delta$-injective right $R$-module with $S=\operatorname{End}\left(Q_{R}\right)$. If $M$ is a $Q$-finitely generated (in particular, finitely generated) right $R$-module, then ${ }_{S} \operatorname{Hom}_{R}(M, Q)$ has finite length.

COROLLARY 4.8. Let $Q$ be a quasi-injective, $Q$-finitely generated (in particular, finitely generated) right $R$-module such that $R$ is both $Q$-noetherian and $Q$-artinian. Then $S=\operatorname{End}\left(Q_{R}\right)$ is a left artinian ring. In particular, if $Q$ is a $Q$ finitely generated (in particular, finitely generated) $\Delta$-injective right $R$-module, then $S=\operatorname{End}\left(Q_{R}\right)$ is a left artinian ring. 
REMARK. The latter part of Corollary 4.8 is due to Faith [3, Corollary 6.4] and Năstăsescu [10, Proposition 1.5].

THEOREM 4.9. Let $U$ be a right $R$-module such that $R$ is both $U$-noetherian and $U$-artinian. If $Q$ is a quasi-injective, $U$-torsionless, $U$-finitely generated right $R$-module such that $U$ is $Q$-injective, then $S=\operatorname{End}\left(Q_{R}\right)$ is a left artinian ring.

ProOF. Since $Q$ is a $U$-finitely generated right module over a $U$-noetherian and $U$-artinian ring $R$ and since $U$ is $Q$-injective, then $Q_{R}$ is both $U$-noetherian and $U$-artinian by Corollary 3.5. So $C_{U}(Q)$ is a noetherian and artinian lattice by Lemmas 3.2 and 3.3. On the other hand, since $Q_{R}$ is $U$-torsionless, every $Q$-closed submodule of $Q$ is also a $U$-closed submodule of $Q$. Hence $C_{Q}(Q)$ is a noetherian and artinian lattice, too. Thus, since $Q_{R}$ is quasi-injective, $Q_{R}$ has a $Q$-composition series by [6, Theorem 2.6]. Therefore, according to [6, Theorems 2.8 and 3.4], we have $\operatorname{len}_{S} S=Q-\operatorname{len} Q_{R}=n$ for some integer $n \geq 0$, as desired.

Corollary 4.10 (NAstÁsescu [10, Proposition 1.5]). Let $U$ be a $\Delta$-injective right $R$-module. If $Q$ is a quasi-injective, $U$-torsionless, $U$-finitely generated right $R$-module, then $S=\operatorname{End}\left(Q_{R}\right)$ is a left artinian ring.

Proof. Since $U_{R}$ is $\Delta$-injective, $R$ is a both $U$-noetherian and $U$-artinian ring according to Miller-Teply's theorem in [7]. Hence the result follows directly from Theorem 4.9 .

LEMMA 4.11. Let $Q$ be a quasi-injective right $R$-module such that $R$ is $Q$ artinian, and let us put $T=\operatorname{Biend}\left(Q_{R}\right)$. Then $T$ is a semi-primary ring and $Q_{T}$ is a $\Delta$-injective module.

ProOF. In this case $Q_{R}$ is $\Delta$-quasi-injective by Lemma 3.3. Hence ${ }_{S} Q$ has finite length according to [4, Proposition 8.1], where $S=\operatorname{End}\left(Q_{R}\right)$. Therefore $T=\operatorname{End}(s Q)$ is a semi-primary ring. And, since $Q_{R}$ is finendo and quasiinjective, $Q_{T}$ is injective (refer to [3, Proposition 19.18]). Since $S=\operatorname{End}\left(Q_{T}\right)$, it follows that $Q_{T}$ is $\Delta$-injective by [4, Corollary 7.5].

THEOREM 4.12. If $Q$ is a noetherian, quasi-injective right $R$-module such that $R$ is $Q$-artinian, then we have the following assertions.

(1) $S=\operatorname{End}\left(Q_{R}\right)$ is a left artinian ring.

(2) $T=\operatorname{Biend}\left(Q_{R}\right)$ is a right artinian ring.

Proof. (1) Since $Q_{R}$ is noetherian, so is also $Q_{T}$. In particular, $Q_{T}$ is finitely generated. On the other hand, $Q_{T}$ is $\Delta$-injective by Lemma 4.11, and $S=\operatorname{End}\left(Q_{T}\right)$. Hence $S$ is a left artinian ring by Corollary 4.8 . 
(2) In this case $Q_{R}$ is $\Delta$-quasi-injective, and so ${ }_{s} Q$ has finite length by [4, Proposition 8.1]. In particular, $s Q$ is finitely gnerated. Thus, $Q_{T}$ is a finendo, faithful, injective module. So $Q_{T}$ is compactly faithful by [3, Proposition 19.15], that is $T_{T} \hookrightarrow Q_{T}^{n}$ for some integer $n \geq 1$. Since $Q_{T}$ is noetherian, $Q_{T}^{n}$, and hence $T_{T}$ is noetherian. On the other hand, since $T$ is a semi-primary ring by Lemma $4.11, T$ is a right artinian ring.

THEOREM 4.13. If $Q$ is an artinian, quasi-injective right $R$-module such that $R$ is $Q$-artinian, then we have the following assertions.

(1) $S=\operatorname{End}\left(Q_{R}\right)$ is a left artinian ring.

(2) $T=\operatorname{Biend}\left(Q_{R}\right)$ is a right artinian ring.

Proof. In this case $C_{Q}(R)$ is an artinian lattice by Lemma 3.3. Since $\operatorname{Ann}_{R}(Q)=\bigcap_{x \in Q} \operatorname{Ann}_{R}(x)$, there exist a finite number of elements $x_{1}, x_{2}, \ldots, x_{n}$ $\in Q$ such that $\operatorname{Ann}_{R}(Q)=\bigcap_{i=1}^{n} \operatorname{Ann}_{R}\left(x_{i}\right)$. Hence, if we put $\bar{R}=R / \operatorname{Ann}_{R}(Q)$, $\bar{R}_{R} \hookrightarrow x_{1} R \oplus x_{2} R \oplus \cdots \oplus x_{n} R$. Since $x_{1} R \oplus \cdots \oplus x_{n} R$ is an artinian right $R$-module, $\bar{R}_{R}$ is artinian, too. Hence $\bar{R}$ is a right artinian ring. Since $Q_{R}$ is artinian, $Q_{\bar{R}}$ is an artinian module over a right artinian ring $\bar{R}$. Hence $Q_{\bar{R}}$ is also noetherian by $\left[9\right.$, Corollary 1.3]. Thus, $Q_{R}$ is noetherian. Therefore the results follow directly from Theorem 4.12 .

COROLLARY 4.14 (FAITH-NÃTASESCU). Let $Q$ be a $\Delta$-injective right $R$ module with $S=\operatorname{End}\left(Q_{R}\right)$ and $T=\operatorname{Biend}\left(Q_{R}\right)$. If $Q_{R}$ is either noetherian or artinian, then $S$ is a left artinian ring and $T$ is a right artinian ring.

COROLLARY 4.15. Let $R$ be a right artinian ring. If $Q$ is a noetherian (or an artinian), quasi-injective right $R$-module, then $S=\operatorname{End}\left(Q_{R}\right)$ is a left artinian ring and $T=\operatorname{Biend}\left(Q_{R}\right)$ is a right artinian ring.

\section{Endomorphism rings of quasi-projective, quasi-injective modules}

THEOREM 5.1. If $Q$ is a finitely generated projective, quasi-injective right $R$-module such that $R$ is $Q$-artinian, then $S=\operatorname{End}\left(Q_{R}\right)$ is a left artinian ring.

ProOF. According to Corollary 4.5, $S$ is a left noetherian ring. On the other hand, since $Q_{R}$ is $\Delta$-quasi-injective in this case, $s Q$ has finite length by [4, Theorem 8.1]. Hence $T=\operatorname{End}\left({ }_{S} Q\right)$ is a semi-primary ring. And, since $Q_{T}$ is finitely generated projective and $S=\operatorname{End}\left(Q_{T}\right), S$ is a semi-primary ring, too, by [5, Proposition 4.5]. Hence $S$ is a left artinian ring. 
THEOREM 5.2. Let $R$ be a left noetherian ring. If $Q$ is a finitely generated projective, quasi-injective, finendo right $R$-module, then $S=\operatorname{End}\left(Q_{R}\right)$ is a left artinian ring.

PrOOF. In this case $S$ is a left noetherian ring and ${ }_{S} Q$ is finitely generated. Hence ${ }_{S} Q$ is noetherian. And, since $Q_{R}$ is finendo and quasi-injective, $Q_{T}$ is injective by $\left[3\right.$, Proposition 19.18], where $T=\operatorname{Biend}\left(Q_{R}\right)$. Hence $Q_{T}$ is $\Delta$ injective according to [4, Proposition 8.1]. Thus, $Q_{T}$ is a finitely generated $\Delta$-injective module with $S=\operatorname{End}\left(Q_{T}\right)$. Therefore $S$ is a left artinian ring by Corollary 4.8.

THEOREM 5.3. Let $Q$ be a quasi-projective, quasi-injective, artinian right $R$-module. Then $S=\operatorname{End}\left(Q_{R}\right)$ is a left artinian ring.

PROOF. Since $Q_{R}$ is quasi-projective and artinian, $S$ is a semi-primary ring by [2, Corollary 4.14]. On the other hand, since $Q_{R}$ is quasi-injective and artinian, $S$ is a left noetherian ring by [2, Corollary 4.4.] or [6, Corollary 4.4]. Hence $S$ is a left artinian ring.

THEOREM 5.4. Let $Q$ be a quasi-projective, quasi-injective, noetherian right $R$-module. Then $S=\operatorname{End}\left(Q_{R}\right)$ is a right artinian ring.

Proof. Since $Q_{R}$ is quasi-injective and noetherian, $S$ is a semi-primary ring by [2, Corollary 4.5]. On the other hand, since $Q_{R}$ is quasi-projective and noetherian, $S$ is a right noetherian ring by [2, Corollary 4.12]. Hence $S$ is a right artinian ring.

COROLLARY 5.5. Let $Q$ be a quasi-projective, quasi-injective, noetherian or artinian, right $R$-module such that $R$ is $Q$-artinian. Then $S=\operatorname{End}\left(Q_{R}\right)$ is a left and right artinian ring.

PrOOF. First, suppose that $Q_{R}$ is noetherian. Then $S$ is a right artinian ring by Theorem 5.4 , while $S$ is a left artinian ring by Theorem 4.12. Next, consider the case where $Q_{R}$ is artinian. As has been shown in the proof of Theorem 4.13, $Q_{R}$ is necessarily noetherian. Hence the result is due to the first case.

Note. In connection with Theorems 4.12 and 4.13 , it should be noticed that in general, if $Q$ is a quasi-injective right $R$-module having the right artinian biendomorphism ring $T, Q$ is necessarily injective as a right $T$-module. Indeed, since $Q$ is a faithful right module over a right artinian ring $T, Q_{T}$ is compactly faithful according to a result of Beachy [12, Proposition 1] (see also Vámos [13]). On the other hand, since any quasi-injective module $Q_{R}$ remains quasi-injective 
as a module over $T=\operatorname{Biend}\left(Q_{R}\right), Q_{T}$ is compactly faithful and quasi-injective. Therefore $Q_{T}$ is injective by [3, Proposition 19.15], as desired.

The author would like to express his thanks to the referee who has suggested the above comment.

\section{Addendum}

We are able to strengthen Corollary 4.5. Under the same assumption as in Corollary 4.5 we can conclude that $S=\operatorname{End}\left(Q_{R}\right)$ is a left artinian ring. For, since $R$ is also $Q$-noetherian by [4, Theorem 7.1], it follows by Theorems 4.2 and 4.4 that $S$ is both semi-primary and left noetherian. Hence $S$ is left artinian. Thus, our Corollary 4.8 is needless.

\section{References}

[1] T. Albu and C. Nastðsescu, 'Décompositions primaires dans les catégories de Grothendieck commutatives I', J. Reine Angew. Math. 280 (1976), 172-194.

[2] T. Albu and C. Næstasescu, Relative finiteness in module theory (Marcel Dekker Inc., 1984).

[3] C. Faith, Algebra II : Ring theory (Springer-Verlag, 1975).

[4] C. Faith, Injective modules and injective quotient rings (Marcel Dekker Inc., 1982).

[5] T. Izawa, 'Maximal quotient rings of endomorphism rings of $E\left(R_{R}\right)$-torsionfree generators', Canad. J. Math. 33 (1981), 585-605.

[6] T. Izawa, 'Composition series relative to a module', J. Pure Appl. Algebra 35 (1985), 15-33.

[7] R. W. Miller and M. L. Teply, 'The descending chain condition relative to a torsion theory', Pacific J. Math. 83 (1979), 207-220.

[8] C. Năstæasescu, 'La structure des modules par rapport à une topologie additive', Tôhoku Math. J. 26 (1974), 173-201.

[9] C. Nastðasescu, 'Conditions de finitude pour les modules', Rev. Roumaine Math. Pures Appl. 24 (1979), 745-758.

[10] C. Nðstăsescu, 'Conditions de finitude pour les modules II', Rev. Roumaine Math. Pures Appl. 25 (1980), 615-630.

[11] C. Năstæasescu and C. Niţă, 'Objets noethérian par rapport à une sous-catégorie èpaisse d'une catégorie abélienne', Rev. Roumaine Math. Pures Appl. 9 (1965), 1459-1468.

[12] J. A. Beachy, 'On quasi-artinian rings', J. London Math. Soc. (2) 3 (1971), 449-452.

[13] P. Vámos, "The dual of the notion of "finitely generated" ', J. London Math. Soc. 43 (1968), 643-646.

Department of Mathematics

Shizuoka University

Ohya 836, Shizuoka 422

Japan 